\title{
Shape resonance for the anisotropic superconducting gaps near a Lifshitz transition: the effect of electron hopping between layers
}

\author{
Davide Innocenti, Sergio Caprara, Nicola Poccia, Alessandro Ricci, Antonio Valletta ${ }^{+}$, and Antonio Bianconi \\ Physics Department, Sapienza University of Rome, Piazzale Aldo Moro 2, 00185 Rome, Italy and \\ ${ }^{+}$Institute for Microelectronics and Microsystems, IMM CNR, \\ Via del Fosso del Cavaliere 100, 00133 Roma, Italy
}

(Dated: November 2, 2018)

\begin{abstract}
The multigap superconductivity modulated by quantum confinement effects in a superlattice of quantum wells is presented. Our theoretical BCS approach captures the low-energy physics of a shape resonance in the superconducting gaps when the chemical potential is tuned near a Lifshitz transition. We focus on the case of weak Cooper-pairing coupling channels and strong pair exchange interaction driven by repulsive Coulomb interaction that allows to use the BCS theory in the weakcoupling regime neglecting retardation effects like in quantum condensates of ultracold gases. The calculated matrix element effects in the pairing interaction are shown to yield a complex physics near the particular quantum critical points due to Lifshitz transitions in multigap superconductivity. Strong deviations of the ratio $2 \Delta / T_{c}$ from the standard BCS value as a function of the position of the chemical potential relative to the Lifshitz transition point measured by the Lifshitz parameter are found. The response of the condensate phase to the tuning of the Lifshitz parameter is compared with the response of ultracold gases in the BCS-BEC crossover tuned by an external magnetic field. The results provide the description of the condensates in this regime where matrix element effects play a key role.
\end{abstract}

PACS numbers: 74.62.-c,74.70.Ad,74.78.Fk

\section{INTRODUCTION}

For a long time the conventional theoretical models for high-temperature superconductivity (HTS) in cuprates have been based on a single-component electronic system with a single effective band, while compelling experimental evidence of two bands, resulting from different orbitals, crossing the Fermi level was reported since 1988 $\stackrel{1}{=}$ Unconventional theories for HTS have been proposed focusing on the characteristics of multigap superconductivity ${ }^{2-4}-\frac{4}{-}$ The possible role of a Lifshitz transition ${ }^{5.6}$ [called also electronic topological transition (ETT) or quantum phase transitions (QPT) of the 2.5-order] in HTS was discussed in the frame of the single band model $\stackrel{\underline{7}}{=}$ In 1993 it was proposed a novel paradigm that identifies the quantum mechanism for rising the critical temperature in the high-temperature range: the shape resonance in superconducting gaps in multiband systems where the chemical potential is tuned near a Lifshitz transition in one of the bands $\underline{\underline{8}} \underline{-13}$

In the last ten years the experimental research has been the driving force for shifting the majority of the scientific community toward the novel paradigm that HTS emerges in complex systems with multiple electronic components in the proximity of a Lifshitz transition. In fact two electronic components at the Fermi level have been found by many groups in cuprates, $\underline{14}-19$ showing two (or more) strongly correlated bands with tendency to phase separation 20,21 The two bands in $\mathrm{YBaCuO}$ system observed in $1988^{1}$ are now confirmed by an increasing number of experiments ${ }^{22}-25$ and recent theoretical models consider the new paradigm of a multiband system near a Lifshitz transition $\underline{26,27}$ The theory of multi- band or multigap superconductivity in the clean limit was developed since long ago, but it was accepted only after the discovery of the two-gap superconductivity at high temperature in magnesium diboride $\mathrm{MgB}_{2}{ }^{28}$ and in doped diborides ${ }^{29}-32$ Later on, low-temperature multiband superconductivity has been found in several materials, such as titanates, ruthenates, boro-carbides, selenides. However, the origin of HTS in $\mathrm{MgB}_{2}$ has been assigned by many authors to the strong electron-phonon intraband coupling in the $\sigma$ band. On the contrary the unique features of multigap superconductivity near a Lifshitz transition ${ }^{29,32,33}$ received little attention by the majority of the HTS community. High-temperature multiband superconductivity has been discovered in 2008 in Fe pnictide multilayers. These materials show a clear weak intraband electron-phonon coupling, therefore multigap superconductivity at $50 \mathrm{~K}$ in pnictides has determined in these last two years the gradual shift of the scientific opinion toward the nowadays widely accepted idea that specific features of multigap superconductivity may be essential for the emergence of HTS $\stackrel{34-36}{-36}$ Recently, the search for HTS is focusing on the control of multiband superconductivity in carbon nanotubes,,$\frac{37}{3}$ graphene bilayers,, 38 and graphane $\underline{39}$

Looking for common features of different high- $T_{c}$ superconducting materials (e.g., cuprates, diborides and pnictides), one could list the following: first, multigap superconductivity; $\stackrel{40}{\longleftarrow}$ second, multiple electronic components, $\stackrel{41}{=}$ third, a heterostructure at the atomic limit, made of superconducting atomic layers intercalated by spacers made of a different material, forming a superlattice ${ }^{8.9}$ as shown in Fig. 1; fourth, the tuning of the Fermi energy (using any physical, chemical or mate- 
rial manipulation method, such as doping and superlattice misfit strain) in order to reach a particular point in the electronic band structure $\stackrel{42-45}{\underline{45}}$ These are the features which were proposed to be essential for the synthesis of new high- $T_{c}$ superconductors, with $T_{c}$ eventually reaching room temperature, in Refs. 8-10. The particular condition yielding high $T_{c}$ was thereby identified as the tuning of the Fermi energy at a shape resonance for the superconducting gaps near a Lifshitz transition. In this work we first discuss the fundamental points of shape resonances in multiband superconductors made of a first two-dimensional (2D) cylindrical Fermi Surface and a second Fermi surface, tuning the chemical potention near the Lifshitz transition. We present the results for the a system of weak intraband coupling so the system is well described by the BCS weak-coupling limit and we consider a case where the interband pairing is the dominant interaction so that it could describe the case of pnictides where the interband pairing is the dominant interaction. In this regime we investigate the effect of variable electron hopping between the layers that is the characteristic feature of superlattices of superconducting layers at atomic limit.

\section{SHAPE RESONANCES FOR SUPERCONDUCTING GAPS}

The concept of shape resonance for superconducting gap parameters was first introduced by Blatt and Thompson for a single $2 \mathrm{D}$ membrane $\frac{46}{47}$ and it has been developed for a single nanowire $\stackrel{47}{\underline{4}}$ The shape resonance for the superconducting gaps in superlattices of quantum wells, (quantum layers, quantum wires and quantum dots $)^{8,9,11}$ has been proposed to yield three-dimensional (3D) superconductors. Shape resonances in a superlattice of stripes in cuprates, $\stackrel{48-50}{=}$ and pnictides $\stackrel{36}{ }$, of layers in diborides,,$\frac{33,51-53}{5}$ and in a superlattice of nanowires in carbon nanotubes, have been discussed 37 The novel scenario for a multiband system with a dominant role of interband pairing and negligible intraband coupling has been proposed before the discovery of pnictides.

The definition of shape resonance in the theory of superconductivity $\underline{\underline{46}}$ is borrowed from nuclear physics. Ettore Majorana first in 1929-1930 developed a theoretical model to describe the artificial disintegration of nuclei by bombardment with $\alpha$-particles 55 By following the quantum dynamics of a state resulting from the superposition of a discrete state with a continuum one, whose interaction is described by a given potential term, Majorana has made the first application of the concept of the idea of quasi-stationary states interacting with the continuum $\frac{55,57}{5}$ The theory of shapeelastic scattering cross section for nucleon-nucleus resonances, where the total energy of incoming or outgoing particles and the quasi stationary states of the compound nucleus are nearly degenerate, was developed in the fifties by Blatt, Feshbach and de Shalit $\underline{58-60}$ The

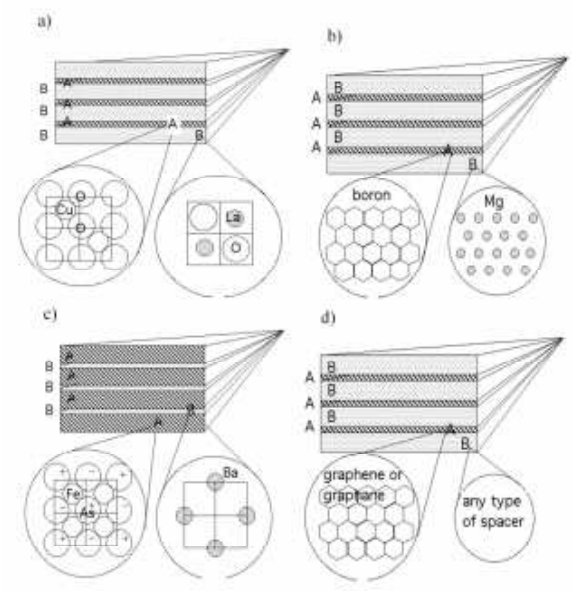

FIG. 1. (Color online). Pictorial view of artificial heterostructures realized as superlattices of stacked planes, with particulars of the superconducting layer and of the spacer layers, in four different families of compounds: (a) cuprates, (b) magnesium diborides, (c) iron pnictides, and (d) graphene or graphane layers.

shape resonance in nuclear scattering from a potential exhibits characteristic peaks, as a function of energy, for values of energy such that an integer number of wavelengths fits within the nuclear potential well. The shape resonances have been measured in electron-atom scattering ${ }^{61}$ and electron-molecule scattering. ${ }^{62,63}$ The name Feshbach resonance was coined to indicate a particular shape resonance, where the quasi-bound state is near zero energy 63 The Feshbach resonance in atomic association and dissociation processes occurs in ultra-dilute and ultra-cold atomic gases where the energy of a diatomic molecule (tuned by an external magnetic field) is degenerate with the chemical potential of the atomic gas $\underline{64}$ In this condition the exchange interaction give the Feshbach resonance. The discovery that in boson and fermion ultracold gases the energy of the diatomic molecule can be tuned (by means of a magnetic field) above and below the continuum threshold has driven to the realization of the Bose-Einstein condensation (BEC) in boson gases and of the BCS-BEC crossover in fermion gases driven by the Feshbach resonance $\underline{65} \underline{67}$ Therefore the shape resonance and Feshbach resonance belong to the class of Majorana-Fano-Feshbach resonances due to configuration interaction effects between open and closed scattering channels described by the Feshbach-Fano partitioning method. $\underline{68}-\underline{\underline{70}}$ In a multiband superconducting system, when the chemical potential is tuned at the Lifshitz transition in one of the bands, the pairs of electrons at the Lifshitz transitions are in a quantum critical point where the group velocity of electrons goes to zero and they form bosonic pairs that can be considered the equivalent of the Majorana quasi-stationary states or the diatomic molecule in the magnetic field in ultra cold 
gases.

In superlattices, the shape resonance ${ }^{11-13,37,53}$ in the superconducting gaps appears when the Fermi energy of electrons in a band is tuned around the Lifshitz transitions $\underline{11}$ in a different band. There are several types of Lifshitz transitions:

type I) when the Fermi energy crosses the band edge, at $E_{\text {edge }}$, with the appearance or disappearance of a new Fermi surface (FS);

type II) when the Fermi energy crosses the electronic topological transition $E_{T} T T_{3 D-2 D}$ where one FS changes from $3 \mathrm{D}$ to $2 \mathrm{D}$ (i.e., from "spherical" to "cylindrical") or vice versa, with the opening or closing of a neck in a tubular FS;

type III) at the singular point where the FS changes from 2D to one-dimensional (1D) or vice versa, with the change of FS topology from a closed FS circle to disconnected Fermi arcs $\stackrel{50}{.}$ Once the heterostructure of materials selected as the building blocks for the superconducting units and for the spacer layers has been synthesized with its structural parameters, the tuning of the Fermi level at an ETT can be controlled by means of: i) the "charge transfer" between the superconducting layers and the "spacers"; ii) the superlattice misfit strain ${ }^{71}-\underline{\underline{73}}$ between the superconducting and spacer layers; iii) the thickness of the spacers; iv) the ordering of dopants in the spacer; $v$ ) the superstructures in the superconducting layers forming stripes; vi) pressure.

\section{BREAKDOWN OF THE STANDARD BCS APPROXIMATIONS}

The heterostructures at the atomic limit of superconducting units, where the chemical potential is tuned at a type I Lifshitz transition (appearing or disappering of a new FS spot) and a the type II Lifshitz transition (opening a neck in a FS), where the superconducting gaps show a shape resonance, have been called superstripes $\underline{\underline{74}}-\underline{\underline{76}}$ From the theoretical point of view, the shape resonance implies the breakdown of two main approximations of the standard BCS theory for superconductivity: 1) the infinite distance of the Fermi energy from the band edges, which is reasonable whenever the energy cutoff for the pairing interaction, $\omega_{0}$, is much smaller than the Fermi energy $E_{F}$ (as measured from the nearest singular point in the electron spectrum): in this case the electron density of states (DOS) can be taken as nearly constant within an energy window of the order of $\omega_{0} ; 2$ ) the single band approximation, which is altogether reasonable in the dirty limit, when impurity scattering mixes the various electron components yielding an effective single-band system.

The situation in which the first approximation breaks down (e.g., when the Fermi energy falls near an electronic topological transition) has been called the van Hove scenario, $\underline{\underline{77}-81}$ or the Pomeranchuk instability scenario,$\underline{82-84}$ belonging to the class of quantum phase

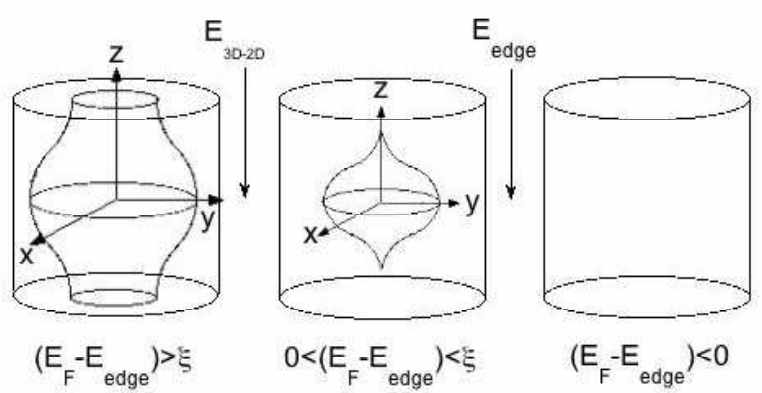

FIG. 2. Pictorial view of the evolution of the FS of the twoband electronic system near a band edge crossing two Lifshitz transitions. The chemical potential is tuned so that $E_{F}$ crosses the band edge $E_{\text {edge }}$ of the second band (the type I Lifshitz transition) and the 3D-2D ETT at $E_{3 D-2 D}$ (the type II Lifshitz transition). Here, $\xi$ is the transversal band dispersion. On the left side a first large 2D FS coexists with a second tubular 2D FS; with decreasing $E_{F}$ and crossing the energy $E_{3 D-2 D}$ where the second FS undergoes a 3D-2D ETT changing its topology, the tubular 2D FS becomes a closed 3D FS as shown in the central panel; only the first large 2D FS remains when the chemical potential is moved below the band edge energy $E_{\text {edge }}$ of the second band as shown in the left panel.

transition scenarios investigated for pairing in single band superconductivity $\stackrel{85-88}{ }$ While these scenarios have been proposed for cuprates assuming a single-band approximation, recently there is increasing evidence for a multiband superconductivity also in cuprates $1,14-20,22-28,41,89,90$

As far as the second approximation is concerned, as we have recalled above, the very concept of multiband superconductivity is meaningful only in the clean limit. Indeed, in nearly all metals and alloys several bands cross the Fermi level, but impurity scattering leads to a mixing of the electron states. Therefore, the singleband description, which is adopted, e.g., in the standard BCS theory, is a reasonable approximation in the dirty limit. However, several experiments in doped diborides have shown that the clean limit is robust in heterostructures at the atomic limit where both the different parity of the bands and their different spatial location forbid the mixing of the different electron states at the Fermi surfaces $\frac{11}{\sim}$ Nowadays it is commonly accepted that the dirty-limit approximation breaks down in magnesium diboride, in pnicitdes, in superlattices of carbon nanotubes, in graphene and graphane layers, and multigap superconductivity may emerge also in the presence of lattice disorder due to dopants and misfit strain of the superlattice, when these are not apt to mix the different electronic components.

Another crucial feature of multiband superconductivity is that, beyond the standard intraband attractive interactions that promote pairing within each band, exchange-like interband interactions, that scatter Coopers pairs from one band to another, become relevant. In 
the hypothetical absence of interband interactions, each band would be characterized by its own superconducting critical temperature. Arbitrarily weak interband interactions lead to a single critical temperature, like in the proximity effect. It is important to notice that interband interactions are generically repulsive and in this case interband pairing leads to condensate wavefunction with opposite signs (the so called $s \pm$ pairing) ${ }^{2-4,11,34}$ In the shape resonance scenario for multiband superconductivity, the control of the ratio between the intensity of exchange-like interband pairing and intraband Cooper pairing, by material design techniques, is crucial.

\section{TWO BAND SUPERCONDUCTIVITY AT A BAND EDGE}

In this work we study the shape resonance in the case when the Fermi energy is tuned at a band edge energy $E_{\text {edge }}$ in one of the bands in a multiband superconductor. In particular we focus on superlattices of metallic layers, with finite hopping probability between the metallic layers. The universal feature of band edges in a superlattice of metallic layers is that the small FS appearing or disappearing as the Fermi energy crosses the band edge has a 3D topology, due to finite electron hopping between the layers separated by a finite potential barrier. We consider the interesting case where the electron hopping between layers is so small that the transversal band dispersion $\xi$ due to hopping between layers is much smaller than the energy separation between the subbands. In these superlattices the FS appearing or disappearing as the Fermi energy crosses the band edge has a 3D topology in the range $0<E_{F}-E_{\text {edge }}<\xi$. When the Fermi energy crosses the van Hove singularity, an $E T T_{3 D-2 D}$ of the Lifshitz type called "opening a neck" takes place, with the FS changing topology, from closed to tubular, at $E_{F}-E_{\text {edge }}=\xi$.

It has been shown 11,36 that the optimum amplification of the critical temperature occurs when $\xi$ is of the order of the energy cut-off of the interaction, $\omega_{0}$. In this case, in the narrow energy range $3 \omega_{0}$ where the chemical potential crosses both the band edge and the $E T T_{3 D-2 D}$, the condensate in the newly appearing FS undergoes a crossover from a mixed Bose-Fermi regime to the Fermi BCS regime. For example, in the regime $-1<\left(E_{F}-E_{\text {edge }}\right) / \omega_{0}<0$, the electron states associated with the newly appearing FS are unoccupied in the normal state, and a BEC-like condensate of pairs with bosonic character is formed below the critical temperature. In the range $0<\left(E_{F}-E_{\text {edge }}\right) / \omega_{0}<1$, all few electrons in the newly appearing $3 \mathrm{D}$ FS in the normal state, condense below $T_{c}$, with the breakdown of the standard approximation $\left(E_{F}-E_{\text {edge }}\right) / \omega_{0} \gg 1$. In the range $0<\left(E_{F}-E_{\text {edge }}\right) / \omega_{0}<1$, in the case under discussion $\xi=\omega_{0}$, the new FS has a $3 \mathrm{D}$ topology. The $E T T_{3 D-2 D}$ plays a key role in the configuration interaction between pairing channels in different bands in the range $0<\left(E_{F}-E_{\text {edge }}\right) / \omega_{0}<2$.

We are interested in the evolution of superconducting gaps in the two bands and of the critical temperature in the narrow energy range where the Feshbach-like shape resonance in superconducting gaps takes place. So, we consider a first large 2D FS in the standard BCS approximation (i.e., where the standard BCS approximation is valid, large $E_{F} / \omega_{0}$ ratio, i.e., $E_{F}$ far from band edges) that coexists with a second appearing small FS, where the standard approximation breaks down (small $E_{F} / \omega_{0}$ ratio, with $E_{F}$ close to both the band edge and the $\left.E_{3 D-2 D}\right)$, see Fig. 2.

This simple model is apt to capture the physics emerging from experiments in doped diborides,, 33 cuprates, 26.27 and pnictides. ${ }^{91-97}$ Moreover it represents the optimal case to obtain the enhancement of critical temperature via the shape resonance mechanism. ${ }^{11}$ It describes the multiband pairing at the bottom or top of $2 \mathrm{D}$ bands in a generic superlattice of layers. Here, the effective potential barrier and the thickness of the spacer layers are selected to adjust the electron hopping between superconducting layers and the transversal energy dispersion $\xi$ of the order of the energy cut-off $\omega_{0}$ of the interaction.

The shape resonance is determined by the relative strength of the intraband coupling constants and the interband coupling constant determined by the type of material forming the superconducting layers and the type of material forming the spacers. We consider here the case, typical of pnictides, where the intraband attractive coupling strength is weak in both the first and second subband. We have obtained the evolution of the superconducting gaps from below the edge, in the mixed Fermi-Bose regime, to above the ETT in the Fermi-like regime. The direct evidence for the quantum interference effect between pairing channels is provided by minima in the gap parameter for electrons in the large FS. We report the evolution of this scenario with increasing exchange interband pairing. We also investigate the effect of the variation of the transversal dispersion $\xi$ that can be changed by changing the separation space between layers. These results can explain the differences among 1111 pnictides, with changing the rare earth ionic radius, and the difference between 1111 and 122 pnictides, where the spacer layers changes in the superlattice, while the superconducting layers are kept fixed.

Finally, we show that the plots of the BCS gap ratios versus the critical temperature that display significant changes in the different superconducting regimes of the Feshbach-like shape resonance. These plots can be easily compared with experimental data and provide a very good experimental test for the theory of the Feshbach-like shape resonance.

There is a clear analogy between the Bose-Fermi crossover case studied here and the BEC-BCS crossover in ultracold Fermi gases ${ }^{98}$ The theory presented here follows Blatt's approach ${ }^{46}$, and it is appropriate for dealing with the case of a chemical potential near a band edge since the equation for the gaps is solved together with 


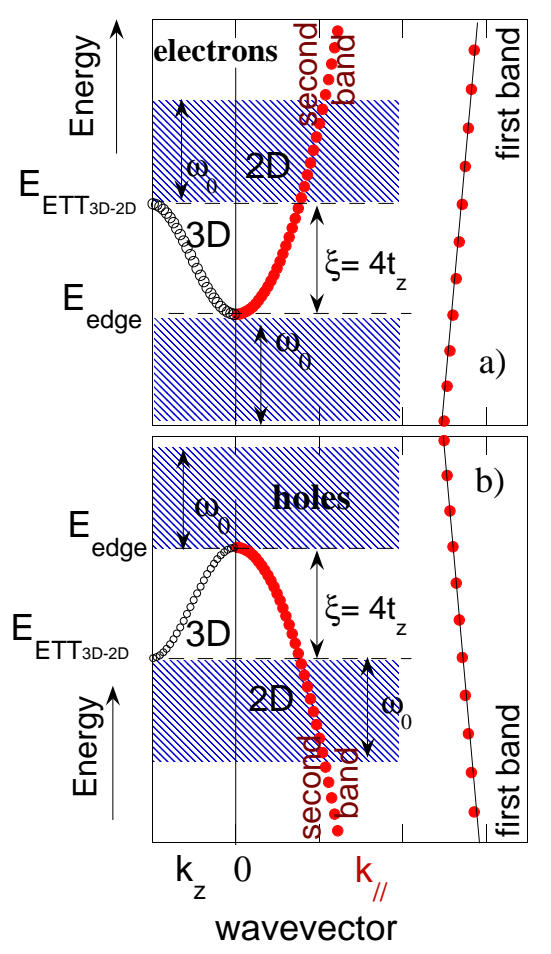

FIG. 3. (Color online). The shape resonance occurs by tuning the Fermi energy near the band edge $E_{\text {edge }}$ of a second electron-like band (upper panel) or a second hole-like band ( lower panel). The second band coexists with a large first band (with the Fermi energy far from its edges) that has a freeelectron like dispersion. The second band has a free-electron dispersion in the direction of the electron wavevector parallel to the plane $\left(k_{/ /}\right)$while in the perpendicular direction $\left(k_{z}\right)$ of the superconducting layers it is determined by a periodic potential barrier determined by the superlattice. This second band has a $3 \mathrm{D}$ character in the energy range between the band edge, and the van Hove singularity energy $E_{E T T_{3 D-2 D}}$. The band dispersion is $\xi=4 t_{z}$, where $t_{z}$ is the electron hopping integral between the layers.

the equation for the chemical potential in the superfluid phase. In fact like for the BCS-BEC crossover, as shown by Leggett $\underline{99}$ the BCS wave function corresponding to an ensemble of overlapping Cooper pairs at weak coupling considering a contact interaction (BCS regime) evolves to non-overlapping pairs with bosonic character as the density decreases at the band edge (BEC regime). The BCS theory remains valid in this limit if the BCS equation for the gap is coupled to the equation that fixes the fermion density so that the chemical potential $\mu$ results strongly renormalized below the critical temperature with respect to the Fermi energy $E_{F}$ of the non interacting system.

In ultracold gases the energy of the bound state of the diatomic molecule above or below the continuum is tuned by a magnetic field. In the present case of superlattices the chemical potential can be tuned near the ETT of a narrow band, for example by superlative misfit strain.

\section{THE TWO BAND MODEL IN A SUPERLATTICE OF METALLIC LAYERS}

As pointed out above, the standard multiband BCS theory in the weak-coupling limit (or the Eliashberg theory in the strong-coupling limit) $)^{2-4}$ assumes a DOS which is (at least approximately) constant within a window of thickness $2 \omega_{0}$ around the Fermi energy. This assumption is certainly not valid if the chemical potential is located near a band edge or close to a van Hove singularity of a superlattice of metallic layers.

Near the band edges in the $\ell$-th band, where $\nabla_{\mathbf{k}} E_{\ell}=0$, the energy of an electron can be approximated by a free electron dispersion law $E_{\ell, \mathbf{k}}=E_{\ell}+\left(\mathbf{k}^{2} / 2 m_{\ell}\right)$, where $m_{\ell}$ is the electron effective mass at the band edge. Therefore, one can use the free electron approximation in a narrow region around the band edge, in the case when the band width is much larger than $2 \omega_{0}$, the energy range of interest for pairing. In the case of interest here, however, while this approximation is valid in the $x, y$ plane of the superconducting layers, it is certainly not valid in the $z$ direction, where the dispersion is on the order of $\omega_{0}$. In this case, an anisotropic band with weak dispersion in the $z$ direction and larger dispersion in the $x, y$ plane above the band edge of the second band should be adopted,

$$
E_{2, \mathbf{k}}^{3 D}=E_{2}+E\left(k_{z}\right)+\frac{k_{x}^{2}+k_{y}^{2}}{2 m_{/ /}}
$$

where $E\left(k_{z}\right)$ is the actual energy dispersion in the periodic potential of the superlattice and $m_{/ /}$is the effective mass in the $x, y$ plane. This situation is obtained within a model where a free electron gas is confined in a potential which is periodic in the $z$ direction,

$$
\mathcal{W}(z)=\sum_{n=-\infty}^{n=+\infty} \mathcal{W}_{b}(z-n d),
$$

where $\mathcal{W}_{b}(z)=-V_{b}$ for $|z| \leq L / 2$ and $\mathcal{W}_{b}(z)=0$ for $L / 2<|z|<d / 2, L$ is the width of the confining well and $d$ is the periodicity of the superlattice in the $z$ direction. This periodic potential mimics the phenomenology, e.g., of the pnictides, diborides, and stacks of graphene layers made of a superlattice of stacked planes, as shown in Fig. 1. The confining potential generates a band structure organized in subbands. Each subband has a dispersion in the transversal direction, as shown in Fig. 2, that gives a Fermi surface with 3D topology, with closed isoenergetic surface, near the lower band edge $E_{\text {edge }}=E_{\ell, L}$, and a $2 \mathrm{D}$ character, with isoenergetic surfaces open in the $z$ direction, above some energy threshold $E_{E T T_{3 D-2 D}}=E_{\ell, T}$. The present model is apt to describe quantum interference phenomena between different scattering channels in a large and a small FS which are the object of this work, when the chemical potential $\mu$ of the system is tuned near the bottom of a $\ell$-th subband, within a window of width $4 \omega_{0}$. In the energy window of width $2 \omega_{0}$ the first band, with a large 2D tubular FS, has a constant DOS 
$N_{1}$. The second FS appears as the Fermi energy crosses the level $E_{\text {edge }}=E_{\ell, L}$, and changes from closed 3D to tubular 2D topology as the Fermi energy crosses the level $E_{E T T_{3 D-2 D}}=E_{\ell, T}$ as it is shown in Fig. 2. The model for electron-like FS can be easily extended to hole-like FS as shown in Fig. 3. The DOS of the second FS $N_{2}$ near the edge has the typical 3D behavior.

In order to solve the BCS equations in the shape resonance scenario it is necessary to determine the electron wavefunction. Using the model of a free electron gas confined in a superlattice, the wavefunction of electronic states can be obtained by solving the Schröedinger equation, so that we can calculate the anisotropic k- dependent gaps in the wavevector space and the interference between the intra-band pairing channels and the pair exchange determined by the interband pairing terms. The solution of the Schröedinger equation for the 1D periodic potential of the superlattice of layers allows us to calculate the gaps also in correspondence of the $3 \mathrm{D}$ to $2 \mathrm{D}$ electronic transition $E T T_{3 D-2 D}$ at the van Hove singularity, beyond the standard BCS approximations $\underline{46,50}$

Going from a single slab $\underline{46}$ to a $3 \mathrm{D}$ superlattice $\frac{50}{}$, a 3D condensate is formed, reducing the effect of fluctuations of the superconducting order parameter, which suppress the mean-field superconducting $T_{c}$. The crossover from $2 \mathrm{D}$ to $3 \mathrm{D}$ can be described in our model by changing from an infinite potential barrier between the planes of the superlattice in the $z$ direction, provided by spacer layers, to a finite barrier $\left(V_{b}\right)$. This yields a finite hopping term that broadens the sharp discontinuity of the DOS of a pure 2D band. This broadening increases the width of the shape resonance and, at the same time, yields a $3 \mathrm{D}$ condensate. Moreover, it is possible to design artificial superlattice heterostructures at optimum shape resonance condition, i.e., where the value of the potential barrier $V_{b}$ and its width are such that the subband dispersion in $z$ direction $\xi$ is of the order of energy cut-off $\omega_{0}$ of the interaction. Therefore, it is possible to tune the system to the optimum shape resonance condition, achieving an enhancement of the critical temperature.

The intrinsic $\mathbf{k}$-dependence of the pairing interaction $\mathcal{V}_{\mathbf{k}, \mathbf{k}^{\prime}}^{\ell, \ell^{\prime}}$ in the superlattice with wave vector $\mathbf{k}_{z}$ induces a structure in the $\mathbf{k}$-dependent interband coupling interaction for the electrons that determines the quantum interference between electron pairs wavefunction in different subbands of the superlattice 11 We calculate the term $\mathcal{V}_{\mathbf{k}, \mathbf{k}^{\prime}}^{\ell, \ell^{\prime}}$ following our previous work for a superlattice of wires, 50 determining the matrix elements of local interaction potential and then introducing a cutoff with two $\theta$ functions,

$$
\mathcal{V}_{\mathbf{k}, \mathbf{k}^{\prime}}^{\ell, \ell^{\prime}}=\tilde{\mathcal{V}}_{\mathbf{k}, \mathbf{k}^{\prime}}^{\ell, \ell^{\prime}} \theta\left(\omega_{0}-\left|\xi_{\ell, \mathbf{k}}\right|\right) \theta\left(\omega_{0}-\left|\xi_{\ell^{\prime}, \mathbf{k}^{\prime}}\right|\right)
$$

where $\mathbf{k}=\mathbf{k}_{z}\left(\mathbf{k}^{\prime}=\mathbf{k}_{z}^{\prime}\right)$ is the superlattice wavevector, in the $z$ direction, perpendicular to the planes, of the initial (final) state in the pairing process, and

$$
\widetilde{\mathcal{V}}_{\mathbf{k}, \mathbf{k}^{\prime}}^{\ell, \ell^{\prime}}=\frac{c_{\ell, \ell^{\prime}}}{N_{0}\left(E_{F}\right) V_{3 D}} I_{\mathbf{k}, \mathbf{k}^{\prime}}^{\ell, \ell^{\prime}}
$$

where $N_{0}\left(E_{F}\right)$ is the DOS at $E_{F}$ for a free electron 3D system, $V_{3 D}$ is the volume of the system,

$$
I_{\mathbf{k}, \mathbf{k}^{\prime}}^{\ell, \ell^{\prime}}=-d \int_{d} \psi_{\ell,-\mathbf{k}}(z) \psi_{\ell^{\prime},-\mathbf{k}^{\prime}}(z) \psi_{\ell, \mathbf{k}}(z) \psi_{\ell^{\prime}, \mathbf{k}^{\prime}}(z) d z
$$

and the $\psi_{\ell, \mathbf{k}}(z)$ are the eigenfunctions in the superlattice of quantum wells, normalized so that $\int_{d} d z\left|\psi_{\ell, \mathbf{k}}(z)\right|^{2}=1$. The use of single cut off in two band superconductivity has been justified in detail in ref 100

The dimensionless factor $c_{\ell, \ell^{\prime}}=(-1)^{\delta_{\ell, \ell^{\prime}}} c_{\ell, \ell^{\prime}}^{0}$ assumes positive values for $\ell=\ell^{\prime}$ (intraband Cooper pairing) and negative values for $\ell \neq \ell^{\prime}$ (repulsive exchange-like interband pairing, with $\left.c_{\ell, \ell^{\prime}}=c_{\ell^{\prime}, \ell}\right)$ and measures the relative intensity of intraband and interband pairing strength. In fact, it multiplicates the $\mathbf{k}$-dependent integral and therefore permits to simulate the behavior of different superconductive multilayer compounds controlling the ratio between intensities of intraband and interband pairings.

In order to determine the gaps and chemical potential self-consistenly and to calculate the superconductive $T_{c}$ we use iterative solving methods for the coupled BCS-like equations

$$
\begin{gathered}
\Delta_{\ell, \mathbf{k}}(\mu)=-\frac{1}{M} \sum_{\ell^{\prime}, \mathbf{k}^{\prime}} \frac{\mathcal{V}_{\mathbf{k}, \mathbf{k}^{\prime}}^{\ell, \ell^{\prime}} \Delta_{\ell^{\prime}, \mathbf{k}^{\prime}}}{2 \sqrt{\left(E_{\ell^{\prime}, \mathbf{k}^{\prime}}-\mu\right)^{2}+\Delta_{\ell^{\prime}, \mathbf{k}^{\prime}}^{2}}}, \\
\rho=\frac{1}{d^{2}} \sum_{\ell, \mathbf{k}}\left[1-\frac{E_{\ell, \mathbf{k}}-\mu}{\sqrt{\left(E_{\ell, \mathbf{k}}-\mu\right)^{2}+\Delta_{\ell, \mathbf{k}}^{2}}}\right],
\end{gathered}
$$

starting with an initial gap parameter equal to a constant and an initial chemical potential equal to the Fermi level in the normal state and considering the convergence occurred for relative variation of the gap and charge density $\rho$ less than $10^{-6}$. Here $M$ is the total number of wavevectors $\mathbf{k}^{\prime}$ and $\rho$ is the electron density. The superconducting critical temperature $T_{c}$ is calculated by iterativly solving the linearized equation

$$
\Delta_{\ell, \mathbf{k}}=-\frac{1}{2 M} \sum_{\ell^{\prime}, \mathbf{k}^{\prime}} \mathcal{V}_{\mathbf{k}, \mathbf{k}^{\prime}}^{\ell, \ell^{\prime}} \frac{\tanh \left(\frac{E_{\ell^{\prime}, \mathbf{k}^{\prime}}-\mu}{2 T_{c}}\right)}{E_{\ell^{\prime}, \mathbf{k}^{\prime}}-\mu} \Delta_{\ell^{\prime}, \mathbf{k}^{\prime}}
$$

until the vanishing solution is reached with increasing temperature. Here, the gaps depend on the superlattice wavevector $\mathbf{k}$ as well as on the subband index. Hence, $T_{c}$ and the gap at a given point of $\mathbf{k}$-space become implicit function of all the different values of the gaps in the entire k-space.

In the standard BCS theory, where the Fermi energy is far from the band edges, the relative variation of the chemical potential going from the normal to the superconducting state is expected to be negligible. This is not true when the chemical potential is tuned near the band edge of the second band. In fact, our calculation yields a significant variation of the chemical potential in 


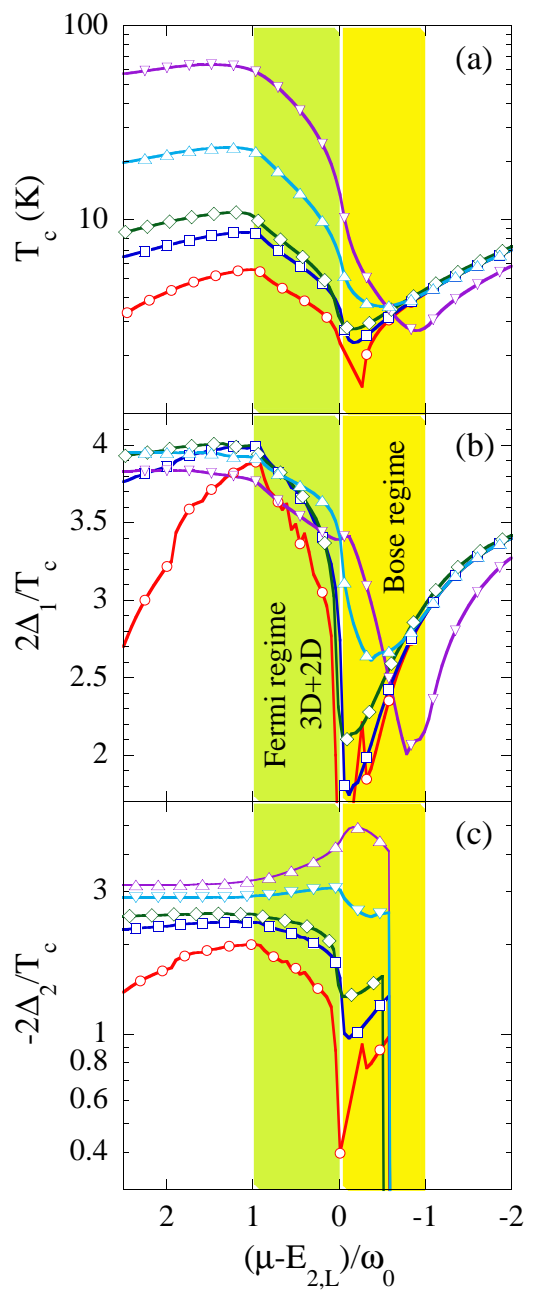

FIG. 4. (Color online). The case of weak coupling in the second band (the so called pnictide case) where the intraband coupling parameters ratio is fixed at $c_{2,2} / c_{1,1}=0.45$. The critical temperature $T_{c}$ (panel a), the ratio $2 \Delta_{1} / T_{c}$ (panel b) and the ratio $-2 \Delta_{2} / T_{c}$ (panel c) are plotted as function of the reduced Lifshitz parameter $\left(\mu-E_{2, L}\right) / \omega_{0}$. The different curves in each panel represent the cases of different interband pairing strength: the case for $c_{12} / c_{11}=-2.73$ (violet curve with open triangles); the case for $c_{12} / c_{11}=$ -1.59 (light blue curve with open triangles); the case for $c_{12} / c_{11}=-1.04$ (green curve with open diamonds); the case for $c_{12} / c_{11}=-0.91$ (dark blue curve with open squares) and shows the case for $c_{12} / c_{11}=-0.68$ (red curve with open circles). For the largest case of interband pairing strength $c_{1,2}$ considered here, the critical temperature reaches $300 \mathrm{~K}$ in the range $1<\left(\mu-E_{2, L}\right) / \omega_{0}<2$. The shape resonance antiresonance appears as a minimum in the critical temperature for $\left(\mu-E_{2, L}\right) / \omega_{0}=-1$ for higher interband repulsive interaction and moves towards $\left(\mu-E_{2, L}\right) / \omega_{0}=0$ for the smaller interband repulsive interaction. The ratio $2 \Delta_{1} / T_{c}$ for the first band shows maxima and minima like the critical temperature. On the contrary the ratio $2 \Delta_{2} / T_{c}$ shows a minimum at $\left(\mu-E_{2, L}\right) / \omega_{0}=0$ and it has a maximum at the value of maximum $T_{c}$ for weak interband repulsive coupling, on the contrary it exhibits a maximum in the Bose-like regime(yellow region) $\left(0>\mu-E_{2, L}\right) / \omega_{0}>-1$ and a minimum value in correspondence of the maximum $T_{c}$ for very strong interband repulsive pairing. the superconducting phase, as a function of the charge density. A relative variation of the chemical potential going from the normal to the superconducting phase, as large as $10^{-3}$, is obtained near the band edge and at the $E T T_{3 D-2 D}$, within a range $4 \omega_{0}$. The variation starts to be large, as compared with the standard BCS result, in proximity of the Bose-Fermi crossover regime below the band edge up to well beyond the $E T T_{3 D-2 D}$.

The Feshbach-like shape resonance regime occurs properly in correspondence of this large variation of the chemical potential between the normal and superconducting phases. Our theoretical approach provides a direct measure of the gap, at a given point of $\mathbf{k}$-space anisotropy for both intraband and exchange interband terms. The intraband distributions of the two bands show different shapes and widths and have different range of values. The resulting matrix of coupling terms is obtained exclusively from the eigenfunctions of the superlattice and is asymmetric.

Below, we present numerical results for the solution of the self-consistency equations which determines the values of the gap in the large FS and in the small FS. We discuss the behavior of $\Delta_{1}$ and $\Delta_{2}$, defined as average values of the gaps on the corresponding branches of the Fermi surface, and of the critical temperature $T_{c}$.

We discuss the case where the coupling term in the second band is smaller than in the first band (called here the pnictide-like case). This case allows to emphasize the role of the exchange-like interband pairing and to use the BCS weak limit for interactions for contact interactions, neglecting retardation effects. In the extreme case when the intraband coupling in the second band is zero, the pairs in the second band are formed only thanks to interband exchange-like pairing.

Therefore in this approach we consider the intraband coupling $c_{1,1}$ for the band that does not show the Lifshitz transition, the intraband coupling $c_{2,2}$ for the one that shows the Lifshitz transition and the inteband coupling $c_{1,2}$ for the exchange-like pairing.

Fig. 4 shows the case of weak coupling in the second band where the intraband coupling parameters ratio is fixed at $c_{2,2} / c_{1,1}=0.45$. The critical temperature $T_{c}$ (panels a ), the ratio $2 \Delta_{1} / T_{c}$ (panel b), and the ratio $-2 \Delta_{2} / T_{c}$ (panel c) are plotted as function of the reduced Lifshitz parameter $\left(\mu-E_{2, L}\right) / \omega_{0}$. The different curves in each panel represent the cases of different interband pairing strengths $\left(c_{1,2} / c_{1,1}=-0.68,-0.91,-1.04\right.$, $-1.59,-2.73)$. In correspondence of the largest interband pairing strength examined here, $c_{1,2}=-2.73$, we have obtained critical temperatures as large as $50 \mathrm{~K}$, in the range $1<\left(\mu-E_{2, L}\right) / \omega_{0}<2$. The antiresonance in the first gap appears when the ratio $2 \Delta_{1} / T_{c}$ reaches the minimum value, that can be much smaller than standard $B C S$ value $\left(2 \Delta / T_{c}=3.53\right)$. The antiresonance appears in the range $-1<\left(\mu-E_{2, L}\right) / \omega_{0}<0$ and moves from -1 to zero decreasing the interband pairing. Moreover the second superconducting gap values results to be nonzero even before the lower band edge $E_{2, L}$ is reached. We notice 


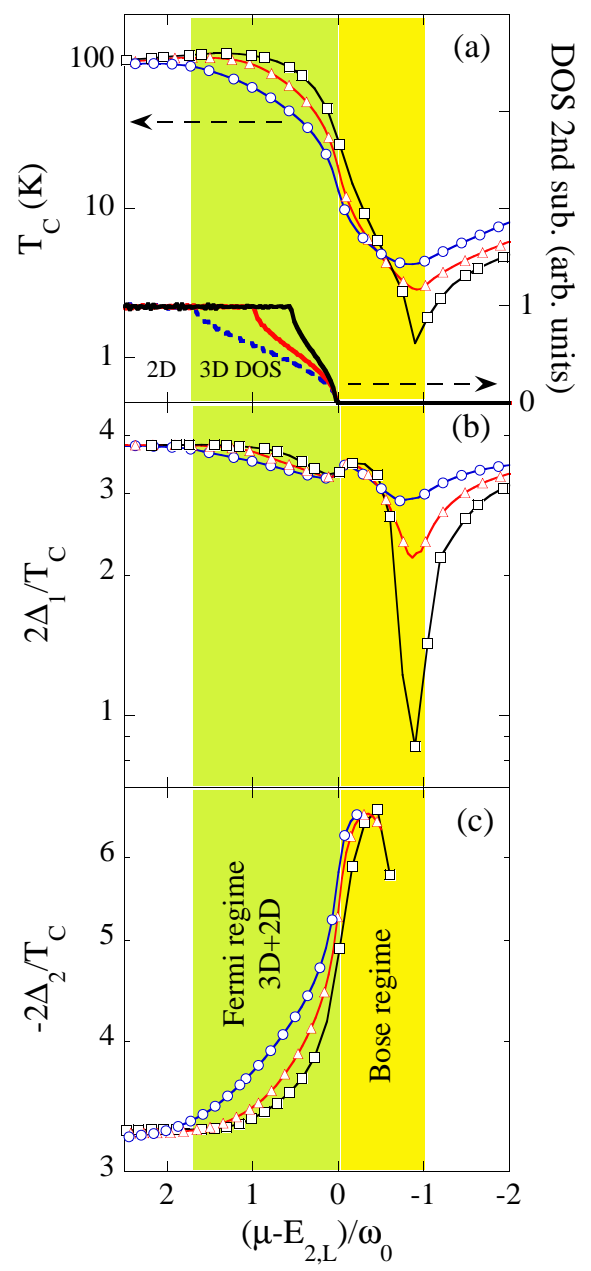

FIG. 5. (Color online). The effect of the variation of transversal dispersion on the shape resonance, the case of weak coupling in the second band (the so called iron pnictide case) for the case of strong interband repulsive interaction. The intraband and interband coupling parameter ratio $\left(c_{2,2} / c_{1,1}=0.45\right.$ and $c_{1,2} / c_{1,1}=-3.6$, with $\left.c_{1,1}=0.22\right)$ are fixed. The critical temperature (panel a), the ratios $2 \Delta_{1} / T_{c}$ (panel b) and $-2 \Delta_{2} / T_{c}$ (panel c) as function of the reduced Lifshitz parameter $\left(\mu-E_{2, L}\right) / \omega_{0}$ are plotted for $\xi=110$ (blue curve with open circles), 68 (red curve with open triangles) and 36 meV (black curve with open squares). This last case is close to the case of a single quantum well. We also plot the DOS of the second subband in the inset of panel (a). The ratio $2 \Delta_{1} / T_{c}$, probing the pairing channel in the first large FS, exhibits two minima due to the negative interference effect (or antiresonance) typical of shape resonances, at the band edge $\left(\mu-E_{2, L}\right) / \omega_{0}=0$ and the the ETT $\left(\mu-E_{2, L}\right) / \omega_{0}=-1$. The antiresonance effect in the large FS increases for decreasing dispersion in the second band. The value of the second superconducting gap is nonzero even before the lower band edge $E_{2, L}$ is reached. In the second band the maximum value of the ratio is always reached in the Bose regime and remains the same, $-2 \Delta_{2} / T_{c} \simeq 6.7$, for all dispersion values. that the maximum $T_{c}$ is reached in the BCS regime zone near the type II Lifshitz transition $\left(E T T_{3 D-2 D}\right)$.

Iron pnictides superconductors are certainly more three dimensional than cuprates (they have a quasi$2 \mathrm{D}$ electronic structure), but the dimensionality of electronic structure in these compounds is also less clear, in fact even if some band structure works predict strong $\mathrm{k}_{z}$ dispersion, $\underline{101}$ other works predict weak dispersions

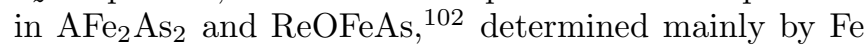
d-orbitals of FeAs layers. Furthermore many angleresolved photoemission spectroscopy experiments $103-108$ reveal weak $\mathrm{k}_{z}$ dependences for the bands electronic structure, in fact most of the band dispersion of 1111 and 11 iron pnictides, like LaOFeAs and NaFeAs,,$\frac{109,110}{,}$ are in the range of $20 \sim 40 \mathrm{meV}$. In 122 undoped pnictides, like $\mathrm{BaFe}_{2} \mathrm{As}_{2}$, the $\mathrm{k}_{z}$ dispersion is rather small, although in $\mathrm{BaFe}_{2-x} \mathrm{Co}_{x} \mathrm{As}_{2}$, with increasing Co concentration, the $\mathrm{k}_{z}$ dispersion increases up to $120 \mathrm{meV} \stackrel{112}{ }$

Fig. 5 shows the effect of the variation of out of plane dispersion on the shape resonance, in the case of weak coupling in the second band (the so called iron pnictide case) for strong interband pairing. The DOS of the second subband for different dispersions $(\xi=110,68$ and $36 \mathrm{meV}$ ), as a function of the reduced Lifshitz parameter $\left(\mu-E_{2, L}\right) / \omega_{0}$, is shown as an inset in panel (a). The intraband and interband coupling parameter ratio $\left(c_{2,2} / c_{1,1}=0.45\right.$ and $c_{1,2} / c_{1,1}=-3.6$, with $\left.c_{1,1}=0.22\right)$ are fixed. The critical temperature, panel (b), the ratios $2 \Delta_{1} / T_{c}$, panel (c), and $-2 \Delta_{2} / T_{c}$, panel (d), are plotted as a function of the reduced Lifshitz parameter $\left(\mu-E_{2, L}\right) / \omega_{0}$. The minima of the ratio $2 \Delta_{1} / T_{c}$, probing the pairing channel in the first large Fermi surface show two minima due to the negative interference effect or antiresonance typical of shape resonances at the band edge $\left(\mu-E_{2, L}\right) / \omega_{0}=0$, and the $\operatorname{ETT}\left(\mu-E_{2, L}\right) / \omega_{0}=-1$. The antiresonance effect in the large FS increases for decreasing dispersion in the second band. The value of the second superconducting gap is nonzero even before the lower band edge $E_{2, L}$ is reached. In the second band, the maximum value of the ratio is always reached in the Bose regime and remains the same, $-2 \Delta_{2} / T_{c} \simeq 6.7$, for all dispersion values.

Using STM spectroscopy it is now possible to measure the gaps and the critical temperature in the same set of experiments. Therefore the BCS gap ratios $2 \Delta_{1} / T_{c}$ and $-2 \Delta_{2} / T_{c}$ as functions of the ratio $T_{c} / T_{c}^{\max }$ can be measured directly for different samples of different gate voltages tuning the chemical potential. The present theory is able to predict these curves and we show that these curves provide a direct measure of the relevance of interband coupling versus Cooper pairing. In fig. 6 we show the case strong interband repulsive interaction and weak coupling for Cooper pairing. We keep constant the ratio $c_{2,2} / c_{1,1}=0.45, c_{1,1}=0.22$ and $\xi / \omega_{0}=1$ and we present the expected behavior of these curves for strong interband interaction. The BCS Gap ratio parameters are plotted for three cases of different interband coupling parameters. When the interband repulsive term is domi- 

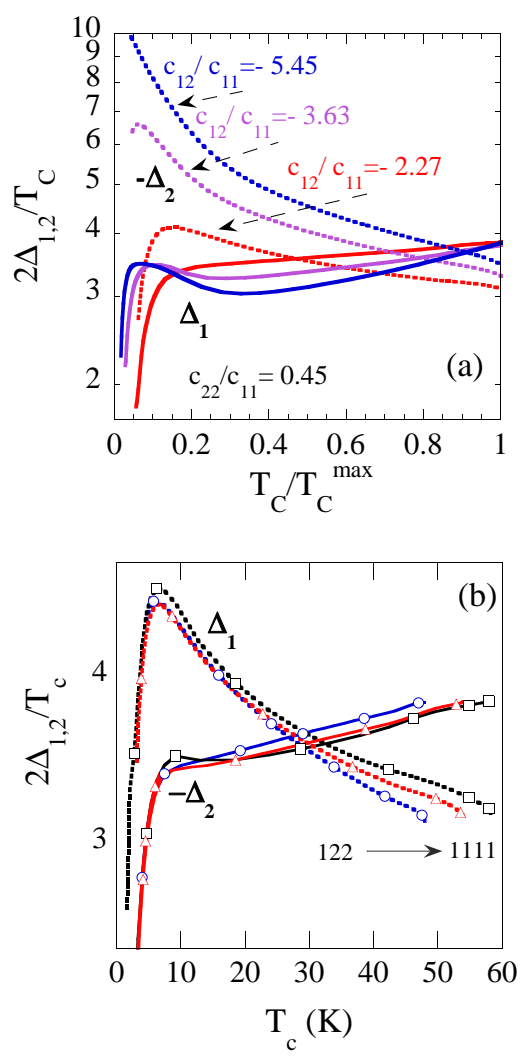

FIG. 6. (Color online). (a) The ratio $2 \Delta_{1} / T_{c}$ and $-2 \Delta_{2} / T_{c}$ as function of the ratio $T_{c} / T_{c}^{\max }$, in the case of weak coupling in the second band, with fixed $c_{2,2} / c_{1,1}=0.45, c_{1,1}=0.22$ and interlayer dispersion $\left(\xi / \omega_{0}=1\right)$, for three cases of different interband coupling parameters $\left(c_{1,2} / c_{1,1}=-2.27,-3-63\right.$, and $-5.45)$ ratio. (b) The ratios $2 \Delta_{1} / T_{c}$ and $-2 \Delta_{2} / T_{c}$ as functions of $T_{c}$ in the case of weak coupling in the second band with fixed $c_{2,2} / c_{1,1}=0.45, c_{1,1}=0.22$ and interlayer dispersion $\left(\xi / \omega_{0}=1\right)$, and in the case of weak coupling in the second band with fixed $c_{1,2} / c_{1,1}=0.45, c_{1,1}=0.22$ and interlayer dispersion $\xi=110$ (blue curve with open circles), 68 (red curve with open triangles) and $36 \mathrm{meV}$ (black curve with open squares).

nant, the BCS gap ratio is the same in both bands at the maximum critical temperature. The difference between the gap ratios diverges decreasing the critical temperature. This behavior is very similar to case of cuprates in fact in the underdoped region where the critical temperature goes to zero and while a first BCS-like gap decreases with the decreasing critical temperature, the second gap (called pseudogap) increases by decreasing the critical temperature.

We have investigated the effect of the band dispersion for a case on moderate relevance of the interband pairing such that there is a characteristic critical temperature where the two gaps are the same and near the standard BCS gap parameter 3.53. We can see that going from the case where the band dispersion is low, i.e., we are close to the regime for a single isolated slab of Blatt to the regime where the dispersion is as large as twice the energy cutoff of the interaction there minor variations. This result shows that the shape resonance due to quantum confinement is a robust feature also for appreciable electron hopping between the quantum wells.

\section{CONCLUSIONS}

The present work provides an interpretation for the properties of quantum size effects in superconducting multilayers and indicate a possible roadmap for the discovery of novel HTS like graphene bilayers and graphane superlattices that should share similarities with the known multigap HTS families where the shape resonance is driven by the interband pairing mechanisms.

Here, we have investigated the effect of electron hopping between quantum wells that can be changed by changing the thickness of spacer layers, like in 1111 pnictides where the rare earth ionic radius in the spacer layer can be changed, or by changing the type of material forming the spacers like going from 1111 to 122 pnictides. To take into account this effect on the shape resonance, we changed the dispersion $\xi$, while keeping it of the order of the pairing interaction energy cut-off $\omega_{0}$. We have fixed in our calculation the intraband Cooper pairing much weaker than the exchange-like interband repulsive interaction. This is clearly the case of iron pnictides that exhibit critical temperatures of the order of $50 \mathrm{~K}$ mostly driven by interband pairing. Namely, we have examined the case of intraband and interband coupling parameter ratio $c_{2,2} / c_{1,1}=0.45$ with variable interband repulsive interactions and variable single electron dispersion in the transversal direction.

The ratios $2 \Delta_{1} / T_{c}$ and $-2 \Delta_{2} / T_{c}$ predicted for high- $T_{c}$ iron-based layered compounds and cuprates as functions of $T_{c}$ show that for the highest $T_{c}$ samples the gaps become equivalent while they are quite different in the low $T_{c}$ samples, that appears to be the scenario for cuprates and for pnictides. The antiresonance typical of shape resonance occurs where the Lifshitz energy parameter is zero (i.e appearing of a new Fermi surface spot) for weak interband exchange pairing and shift to -1 for a very strong interband exchange pairing. The relevant result is that the maximum of the critical temperature appears near the Lifshitz transition of the type opening a neck in corrugated cylindrical surface therefore it moves with the variation of the electronic hopping between the layers i.e., the energy dispersion in the normal direction respect to the superconducting layers.

\section{ACKNOWLEDGMENTS}

We thank Andrea Perali, Ilya Eremin, and Andrei Shanenko for useful discussions. 
1 A Bianconi M De Santis A Di Cicco A M Flank A Fontaine $\mathrm{P}$ Lagarde $\mathrm{H} \mathrm{K}$ Yoshida A Kotani A Marcelli 1988 Physical Review B 387196 URL http://dx.doi.org/10.1103/PhysRevB.38.7196

2 N Kristoffel P Konsin T Ord 1994 Rivista Nuovo Cimento 171

3 NKristoffel P Rubin T Ord 2008 Journal of Physics: Conference Series 108 012034+ ISSN 1742-6596 URL http://dx.doi.org/10.1088/1742-6596/108/1/012034

4 K A Muller A Bussmann-Holder 2005 "Superconductivity in complex systems" (Berlin) Springer-Verlag ISBN 9783-540-23124-0DOI

5 I M Lifshitz 1960 Soviet Physics JEPT 111130 (in Russian in 1960 J Exptl Theoret Phys (U.S.S.R.) 38 1569-1576)

6 A A Varlamov V S Egorov A V Pantsulaya 1989 Adv Phys $38469+$

7 R Markiewicz 1988 Physica C: Superconductivity 153-155 1181-1182

8 A Bianconi 1994 Solid State Communications 89933 ISSN 00381098 URL http://dx.doi.org/10.1016/0038-1098 94 90354-9

9 A Bianconi 1998 'High Tc superconductors made by metal heterostructures at the atomic limit' European Patent No 0733271

10 A Bianconi 2001 'Process of increasing the critical temperature Tc of a Bulk Superconductor by Making Metal Heterostructures at the Atomic Limit' US Patent 6,265,019 B1 URL http://patft.uspto.gov/

11 A Bianconi 2005 Journal of Superconductivity 1825 URL http://dx.doi.org/10.1007/s10948-005-0047-5

12 A Bianconi 2006 Symmetry and Heterogeneity in high temperature superconductors, Nato Science Series II Mathematics, Physics and Chemistry vol 214 Springer Dordrecht The Netherlands ISBN: 10 1-4020-3987-5

13 A Bianconi 2006 Iranian Journal of Physics Research 6 139-147

14 R Khasanov S Strassler D Di Castro $\mathrm{T}$ Masui S Miyasaka S Tajima A B Holder H Keller 2007 Physical Review Letters 99 237601+ URL http://dx.doi.org/10.1103/PhysRevLett.99.237601

$15 \mathrm{~S}$ Hufner M A Hossain A Damascelli G A Sawatzky 2008 Reports on Progress in Physics 71 062501+ ISSN 0034-4885 URL http://dx.doi.org/10.1088/0034-4885/71/6/062501

16 M C Boyer W D Wise K Chatterjee $\mathrm{M}$ Yi Kondo T Takeuchi H Ikuta E W Hudson 2007 Nature Physics $3 \quad 802$ ISSN 1745-2473 URL http://dx.doi.org/10.1038/nphys725

17 D L Feng et al 2002 Physical Review Letters 88 107001+ URL http://dx.doi.org/10.1103/PhysRevLett.88.107001

18 N Doiron-Leyrand et al 2007 Nature 447565

19 E A Yelland J Singleton C H Mielke N Narrison F F Balakirev B Dabrowski J R Cooper 2008 Phys Rev Lett $100047003+$

${ }^{20} \mathrm{~K}$ I Kugel A L Rakhmanov A O Sboychakov N Poccia A Bianconi 2008 Physical Review B 78 165124+ ISSN 1098-0121 URL http://dx.doi.org/10.1103/PhysRevB.78.165124

21 DInnocenti ARicci NPoccia GCampi MFratini ABianconi 2009 Journal of Superconductivity and Novel Magnetism 22529 ISSN 1557-1939 (Print) 1557-1947 (Online) URL http://dx.doi.org/10.1007/s10948-009-0474-9

22 N Doiron-Leyraud et al 2007 Nature 447 565-568 URL http://dx.doi.org/10.1038/nature05872

$23 \mathrm{~J}$ Singleton et al 2010 Physical Re$\begin{array}{lllll}\text { view } & \text { Letters } & 104 & 086403+ & \text { URL }\end{array}$ http://dx.doi.org/10.1103/PhysRevLett.104.086403

24 S E Sebastian et al 2010 Physical Review B $81214524+$ URL http://dx.doi.org/10.1103/PhysRevB.81.214524

${ }^{25} \mathrm{M}$ Norman 2010 Physics $3 \quad 86+\quad$ URL http://dx.doi.org/10.1103/Physics.3.86

26 S G Ovchinnikov M M Korshunov E I Shneyder 2009 Journal of Experimental and Theoretical Physics 1095 775785 URL http://dx.doi.org/10.1134/S1063776109110077

27 M R Norman J Lin A J Millis 2010 Phys Rev B 81 $180513+$

${ }^{28}$ S Tsuda T Yokoya T Kiss Y Takano K Togano H Kito H Ihara S Shin 2001 Physical Review Letters 87 177006+ URL http://dx.doi.org/10.1103/PhysRevLett.87.177006

29 A Bussmann-Holder and A Bianconi 2003 $\begin{array}{llllll}\text { Physical Review } & \text { B } & 67 & 132509+ & \text { URL }\end{array}$ http://dx.doi.org/10.1103/PhysRevB.67.132509

30 S Tsuda et al 2005 Phys Rev B 72064527

${ }^{31}$ L D Cooley A J Zambano A R Moodenbaugh R F Klie Jin-Cheng Zheng and Yimei Zhu 2005 Phys Rev Lett 95 267002

32 D Innocenti N Poccia A Ricci A. Valletta S Caprara A Perali and A. Bianconi 2010 Physical Review B 82 184528+ URL http://dx.doi.org/10.1103/PhysRevB.82.184528

33 A Bianconi D Di Castro S Agrestini G Campi N L Saini A Saccone S D Negri M Giovannini 2001 Journal of Physics: Condensed Matter 137383 URL http://arxiv.org/abs/cond-mat/0103211

$34 \mathrm{C}$ T Chen C C Tsuei M B Ketchen Z A Ren Z X Zhao 2010 Nature Physics 6260 ISSN:1745-2473 URL http://dx.doi.org/10.1038/nphys1531

35 K Kuroki S Onari R Arita H Usui Y Tanaka H Kontani H Aoki 2008 Physical Review Letters 101 087004+ URL http://dx.doi.org/10.1103/PhysRevLett.101.087004

36 R Caivano et al 2009 Superconductor Science and Technology 22 014004+ URL http://arxiv.org/abs/0809.4865

37 A Bianconi 2006 Physica Status So-

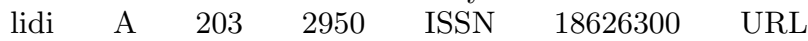
http://dx.doi.org/10.1002/pssa.200567003

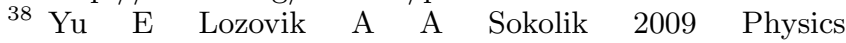
Letters $\quad$ A $374 \quad 326$ ISSN 03759601 URL http://dx.doi.org/10.1016/j.physleta.2009.10.045

39 N Kristoffel K Rago 2010 preprint arXiv:1003.5083 URL http://arxiv.org/abs/1003.5083

40 J Annett F Kusmartsev A Bianconi 2009 Superconductor Science and Technology $22010301+$ URL http://dx.doi.org/10.1088/0953-2048/22/1/010301

41 A Bianconi 2006 Journal of Physics and Chemistry of Solids $67 \quad 567 \quad$ ISSN $00223697 \quad$ URL http://dx.doi.org/10.1016/j.jpcs.2005.10.160

42 A Bianconi N L Saini S Agrestini D Di Castro G Bianconi 2000 International Journal of Modern Physics B 143342 URL http://dx.doi.org/doi:10.1142/S0217979200003812

43 A Bianconi S Agrestini G Bianconi D Di Castro N L Saini 2001 Journal of Alloys and Compounds $\quad 317-318 \quad 537 \quad$ ISSN 09258388 URL 
http://dx.doi.org/10.1016/S0925-8388(00)01383-9

44 A Bianconi N Poccia A Ricci 2009 Journal of Superconductivity and Novel Magnetism 22527 ISSN 1557-1939 URL http://dx.doi.org/10.1007/s10948-009-0471-z

45 A Ricci et al 2010 Physical Review B 82 144507+ URL http://dx.doi.org/10.1103/PhysRevB.82.144507

46 J M Blatt C J Thompson 1963 Phys Rev Lett 10332

47 A A Shanenko M D Croitoru 2006 Physical Review B 73 012510+ URL http://dx.doi.org/10.1103/PhysRevB.73.012510

48 A Bianconi N L Saini T Rossetti A Lanzara A Perali M Missori $\mathrm{H}$ Oyanagi $\mathrm{H}$ Yamaguchi $\mathrm{Y}$ Nishihara D H Ha 1996 Physical Review B 5412018 URL http://dx.doi.org/10.1103/PhysRevB.54.12018

49 A Perali A Bianconi A Lanzara N L Saini 1996 Solid State Communications 100181 ISSN 00381098 URL http://dx.doi.org/10.1016/0038-1098 96 00373-0

50 A Bianconi A Valletta A Perali N L Saini 1998 Physica $\quad$ C $296 \quad 269$ ISSN 09214534 URL http://dx.doi.org/10.1016/S0921-4534 97 01825-X

51 A Bianconi S Agrestini A Bussmann-Holder 2004 Journal of Superconductivity 17205 ISSN 0896-1107 URL http://dx.doi.org/10.1023/B:JOSC.0000021214.52321.ab

52 G G N Angilella A Bianconi R Pucci 2005 Journal of Superconductivity and Novel Magnetism 1819 ISSN 15571939 URL http://dx.doi.org/10.1007/s10948-005-0049-3

53 M Filippi A Bianconi A Bussmann-Holder 2005 Journal de Physique IV Proceedings 13149 ISSN 1155-4339 URL http://dx.doi.org/10.1051/jp4:2005131010

54 J Rodriguez Nunez A Schmidt A Bianconi A Perali 2008 Physica C: Superconductivity 4682299 ISSN 09214534 URL http://dx.doi.org/10.1016/j.physc.2008.05.050

$55 \mathrm{~S}$ Esposito E Recami A van der Merwe R Battiston 2009 Ettore Majorana: Unpublished Research Notes on Theoretical Physic 159 FUNDAMENTAL THEORIES OF PHYSICS S Esposito E Recami A van der Merwe R Battiston (Berlin) Springer ISBN 1402091133 DOI: 10.1007/978-1-4020-9114-8

${ }^{56}$ E DiGrezia S Esposito 2008 Foundations of Physics $38 \quad 228$ ISSN $0015-9018$ URL http://dx.doi.org/10.1007/s10701-007-9200-2

57 AVittorini-Orgeas ABianconi 2009 Journal of Superconductivity and Novel Magnetism 22215 ISSN 1557-1939 URL http://dx.doi.org/10.1007/s10948-008-0433-x

58 J M Blatt V F Weisskopf 1952 Theoretical Nuclear Physics (New York) John Wiley and Sons Inc 401

${ }^{59} \mathrm{H}$ Feshbach C E Porter V F Weisskopf 1954 Physical Review 96 448 URL http://dx.doi.org/10.1103/PhysRev.96.448

60 A de Shalit H Feshbach 1974 Theoretical Nuclear Physics: Nuclear structure 1 (New York) John Wiley and Sons Inc 87

61 Y Hahn T F O'Malley L Spruch 1962 Phys Rev 128932 URL http://dx.doi.org/10.1103/PhysRev.128.932

${ }^{62} \mathrm{~T} \quad \mathrm{~F} \quad$ O'Malley 1966 Phys Rev $150 \quad 14 \quad$ URL http://dx.doi.org/10.1103/PhysRev.150.14

63 J N Bardsley F Mandl 1968 Reports on Progress in Physics $31471+$ ISSN 0034-4885 URL http://dx.doi.org/10.1088/0034-4885/31/2/302

$64 \mathrm{C}$ Chin RGrimm PJulienne E Tiesinga 2010 Reviews of Modern Physics $82 \quad 1225$ URL http://dx.doi.org/10.1103/RevModPhys.82.1225

65 E Tiesinga B J Verhaar H T C Stoof 1993 Physical Review A $47 \quad 4114$ URL
http://dx.doi.org/10.1103/PhysRevA.47.4114

66 S Inouye M R Andrews J Stenger H J Miesner D M Stamper-Kurn W Ketterle 1998 Nature 392151 ISSN 0028-0836 URL http://dx.doi.org/10.1038/32354

67 R Duine H T $\mathrm{C}$ Stoof 2004 Physics Reports $396 \quad 115 \quad$ ISSN 03701573 URL http://dx.doi.org/10.1016/j.physrep.2004.03.003

68 U Fano 1935 Nuovo Cimento 128156 English translation arXiv:cond/mat0502210 10 Feb 2005

${ }^{69} \mathrm{U}$ Fano 1961 Physical Review 1241866 URL http://dx.doi.org/10.1103/PhysRev.124.1866

70 H Feshbach 1958 Ann Phys (New York)5 357

71 S Agrestini N L Saini G Bianconi A Bianconi 2003 Journal of Physics A: Mathematical and General 369133 URL http://dx.doi.org/10.1088/0305-4470/36/35/302

72 M Fratini N Poccia A Bianconi 2008 Journal of Physics: Conference Series 108 012036+ ISSN 1742-6596 URL http://dx.doi.org/10.1088/1742-6596/108/1/012036

73 NPoccia M Fratini 2009 Journal of Superconductivity and Novel Magnetism 22299 ISSN 1557-1939 URL http://dx.doi.org/10.1007/s10948-008-0435-8

74 A Bianconi D Di Castro N L Saini G Bianconi 2002 Phase Transitions and Self-Organization in Electronic and Molecular Networks Fundamental Materials Research chap 24 M F Thorpe J C Phillips Kluwer (Boston) Academic Publishers 375-388 ISBN 0-306-46568-X URL http://dx.doi.org/10.1007/0-306-47113-2-24

75 A Bianconi $2000 \quad$ International Journal of Modern Physics B $14 \quad 3289$ URL http://dx.doi.org/10.1142/S0217979200003769

76 N L Saini A Bianconi 2000 International Journal of Modern Physics B $14 \quad 3649$ URL http://dx.doi.org/10.1142/S0217979200004179

77 J E Hirsch D J Scalapino 1986 Physical Review Letters $56 \quad 2732$ URL http://dx.doi.org/10.1103/PhysRevLett.56.2732

78 J Labbe J Bok 1987 EPL Europhysics Letters $3 \quad 1225+\quad$ ISSN $0295-5075$ URL http://dx.doi.org/10.1209/0295-5075/3/11/012

79 D M Newns C $\quad$ C $\quad$ Tsuei P C Pattnaik 1995 Physical Review $\mathrm{B} \quad 52 \quad 13611$ URL http://dx.doi.org/10.1103/PhysRevB.52.13611

$80 \mathrm{R}$ Markiewicz 1997 Journal of Physics and Chemistry of Solids $58 \quad 1179$ ISSN 00223697 URL http://dx.doi.org/10.1016/S0022-3697 97 00025-5

81 E Cappelluti L Pietronero 1996 Physical Review B 53932 URL http://dx.doi.org/10.1103/PhysRevB.53.932

82 C J Halboth W Metzner 2000 Physical Review Letters $85 \quad 5162$ URL \begin{tabular}{|llll}
\hline http://dx.doi.org/10.1103/PhysRevLett.85.5162 & \\
H & Yamase & W & Metzner 2007
\end{tabular} ical Review $\quad$ B $\quad 75 \quad 155117+\quad$ URL

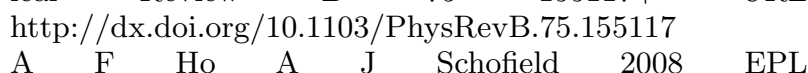
$\begin{array}{lllll}\text { Europhysics } & \text { Letters } & 84 & 27007 & \text { URL }\end{array}$ http://dx.doi.org/10.1209/0295-5075/84/27007

85 E Dagotto 1994 Rev Mod Phys 66763

${ }^{86}$ M Imada A Fujimori Y Tokura 1998 Rev Mod Phys 70 1039

87 S G Ovchinnikov 1997 Phys Usp 40993

88 E G Maksimov 2000 Phys Usp 43965

89 A Bianconi N L Saini A Lanzara M Missori $\mathrm{T}$ Rossetti $\mathrm{H}$ Oyanagi $\mathrm{H}$ Yamaguchi $\mathrm{K}$ Oka $\mathrm{T}$ Ito 1996 Physical Review Letters $76 \quad 3412$ URL 
http://dx.doi.org/10.1103/PhysRevLett.76.3412

90 Saini N L et al 1997 Physical Review Letters 79 3467-3470 URL http://dx.doi.org/10.1103/PhysRevLett.79.3467

91 S Lebegue Z P Yin W E Pickett 2009 New Journal of Physics 11 025004+ ISSN 1367-2630 URL http://dx.doi.org/10.1088/1367-2630/11/2/025004

92 L S Mazov 2009 preprint arXiv:0911.4089 URL http://arxiv.org/abs/0911.4089

$93 \mathrm{H}$ Shishido et al 2010 Physical Review Letters $104 \quad 057008+\quad$ URL http://dx.doi.org/10.1103/PhysRevLett.104.057008

94 G Cao S Jiang C Wang Y Li Z Ren Q Tao J Dai Z.-A Xu 2009 Physica C: Superconductivity ISSN 09214534 URL http://dx.doi.org/10.1016/j.physc.2009.10.141

95 C Wang S Jiang Q Tao Z Ren Y Li L Li C Feng J Dai G Cao Z.-A Xu 2009 EPL Europhysics Letters 86 47002+ URL http://dx.doi.org/10.1209/0295-5075/86/47002

96 J Dai Q Si J.-X Zhu E Abrahams 2009 Proceedings of the National Academy of Sciences 1064118 URL http://dx.doi.org/10.1073/pnas.0900886106

$97 \mathrm{~K}$ Terashima et al 2009 Proceedings of the National Academy of Sciences 1067330 URL http://dx.doi.org/10.1073/pnas.0900469106

98 A Perali P Pieri G C Strinati 2004 Phys Rev Lett 93 100404-1/-4

99 A J Leggett 1980 Modern Trends in the Theory of Condensed Matter Lecture Notes in Physics 115 A Pekalski R Przystawa (Berlin) Springer-Verlag 13
100 P Entel D Rainer 1976 Journal of Low Temperature Physics 23511

101 F Ma Z.-Y Lu T Xiang 2010 Front Phys China 5(2) 150 DOI:10.1007/s11467-009-0076-9

102 I A Nekrasov Z V Pchelkina M V Sadovskii 2008 JETP Lett 882 144-149 DOI:10.1134/S0021364008140166

$103 \mathrm{H}$ Ding et al 2008 Europhys Lett 8347001 DOI:10.1209/0295-5075/83/47001

104 V B Zabolotnyy et al 2009 Physica C: Superconductivity 469 448-451 DOI:10.1016/j.physc.2009.03.043

${ }^{105} \mathrm{C}$ Liu et al 2008 Phys Rev Lett 101177005 DOI:10.1103/PhysRevLett.101.177005

106 L Wray et al 2008 Phys Rev B $78 \quad 184508$ DOI:10.1103/PhysRevB.78.184508

107 C Liu et al 2009 Phys Rev Lett 102167004 DOI:10.1103/PhysRevLett.102.167004

108 T Kondo et al 2008 Phys Rev Lett 101147003 DOI:10.1103/PhysRevLett.101.147003

109 L X Yang et al 2010 Phys Rev B 82104519 DOI:10.1103/PhysRevB.82.104519

${ }^{110} \mathrm{C}$ He et al 2010 Journal of Physics and Chemistry of Solids DOI:10.1016/j.jpcs.2010.10.078

111 S Thirupathaiah et al 2010 Phys Rev B 81104512 DOI:10.1103/PhysRevB.81.104512

112 P Vilmercati et al 2009 Phys Rev B 79220503 DOI:10.1103/PhysRevB.79.220503 